\title{
VIEWPOINT
}

\section{Smartphone-Based Screening for Cardiovascular Diseases: A Trend?}

\author{
Thiago Mazzu-Nascimento, ${ }^{\circledR}$ Danilo Nogueira Evangelista, ${ }^{1 \oplus}$ Obeedu Abubakar, ${ }^{\circledR}$ Meliza Goi Roscani, ${ }^{\circledR}$ \\ Rodrigo Santos Aguilar, ${ }^{1,2}$ Silvana Gama Florêncio Chachá, ${ }^{1 \oplus}$ Priscila Raupp da Rosa, ${ }^{3 \oplus}$ Diego Furtado Silva ${ }^{\circledR}$ \\ Universidade Federal de São Carlos, ' São Paulo, SP - Brazil \\ HU-UFSCar - Hospital Universitário da UFSCar - Ebserh, ${ }^{2}$ São Paulo, SP - Brazil \\ Universidade Nove de Julho - UNINOVE, ${ }^{3}$ São Paulo, SP - Brazil
}

\section{Abstract}

Cardiovascular diseases are the leading cause of death in the world. People living in vulnerable and poor places such as slums, rural areas and remote locations have difficulty in accessing medical care and diagnostic tests. In addition, given the COVID-19 pandemic, we are witnessing an increase in the use of telemedicine and noninvasive tools for monitoring vital signs. These questions motivate us to write this point of view and to describe some of the main innovations used for non-invasive screening of heart diseases. Smartphones are widely used by the population and are perfect tools for screening cardiovascular diseases. They are equipped with camera, flashlight, microphone, processor, and internet connection, which allow optical, electrical, and acoustic analysis of cardiovascular phenomena. Thus, when using signal processing and artificial intelligence approaches, smartphones may have predictive power for cardiovascular diseases. Here we present different smartphone approaches to analyze signals obtained from various methods including photoplethysmography, phonocardiograph, and electrocardiography to estimate heart rate, blood pressure, oxygen saturation $\left(\mathrm{SpO}_{2}\right)$, heart murmurs and electrical conduction. Our objective is to present innovations in non-invasive diagnostics using the smartphone and to reflect on these trending

\section{Keywords}

Smartphone/trends; Artificial Intelligence/trends, Computers System/trends; Cardiovascular Diseases/ diagnóstico; COVID-19/diagnóstico; Smartphone/trends; Diagnostic; Imaging/methods; Telemedicine. approaches. These could help to improve health access and the screening of cardiovascular diseases for millions of people, particularly those living in needy areas.

\section{Introduction}

Cardiovascular diseases (CVD) are the leading cause of death in the world. According to the World Health Organization (WHO), in 2016, it was estimated that 17.9 million people died from conditions related to CVD, representing $31 \%$ of all global deaths. Over $75 \%$ of deaths from CVD occur in low- and middle-income countries. About 6.2 million premature deaths (under the age of 70) from non-communicable diseases are caused by CVD, which include coronary heart disease, cerebrovascular disease, peripheral arterial disease, rheumatic heart disease, congenital heart disease, deep vein thrombosis, and pulmonary embolism. Heart attacks and strokes are the most serious acute events that lead to death. ${ }^{1}$

Increasing researches on technology and communication have been conducted in medicine. Advances in mobile health (mHealth) have helped health professionals in the prevention and early detection of diseases, remote diagnosis, and self-care management, ${ }^{2}$ and significantly reduced the amount of money spent on diagnosing and treating heart diseases.

Telemedicine combines information technology, telecommunication, and data analysis in consultations (teleconsultation), diagnostics (telediagnosis), and robotic surgery (telesurgery). Also, cardiological telemedicine or telecardiology have been expanded, and a wide range of cardiological investigations have been performed remotely, accelerating the flow of information.

Mailing Address: Thiago Mazzu-Nascimento

Rod. Washington Luiz, s/n. Postal Code: 13572-060, São Carlos, São Paulo, SP - Brazil.

E-mail: thiagomazzu@gmail.com 
Electrocardiograms (ECGs), X-ray, ultrasound, and other test results are transmitted for evaluation by specialists (teleconsultants), who provide quick and accurate diagnostics in primary health care (PHC). ${ }^{3}$ The Federal Council of Medicine (CFM) in Brazil indicated the use of digital resources in health and possible introduction of telemedicine during the pandemic, through the letter dated 19th March 2021. ${ }^{4}$

Furthermore, considering the current context of the new coronavirus pandemic (SARS-Cov-2) which causes COVID-19 and the recommendations for social isolation, the use of telemedicine has grown exponentially, ${ }^{5}$ which includes the developement of non-invasive tools for health monitoring of patients with COVID-19. ${ }^{6}$

Countries with a continental territorial dimension, such as Brazil, have great difficulties in providing a comprehensive and decisive service to the entire population. Therefore, telemedicine and mHealth have emerged as opportunities to improve health access for thousands of people, ${ }^{3}$ including underprivileged areas such as slums, rural areas, and remote locations.

In 2018, the number of mobile phones exceeded 5 billion, indicating that almost $70 \%$ of the world population had access to a smartphone. A projection pointed out by the Getúlio Vargas Foundation indicated that in 2018, there were more smartphones than inhabitants in Brazil, with about 220 million devices. ${ }^{7}$

Smartphones are equipped with cameras, flashlights, microphones, processors, internet connection, and function like a computer. Mobile applications have advanced quickly, allowing access to different information about a person's health, from diseases such as anemias, ${ }^{8}$ diabetes, ${ }^{9}$ to cardiovascular conditions. ${ }^{10}$

\section{Measuring vital signs with smartphone}

Vital signs are the first clinical parameters assessed in a health facility. Studies have shown that smartphone applications that use the photoplethysmography (PPG) technique are capable of monitoring heart rate with a quality similar to commercial devices. Smartphone-based photoplethysmography (spPPG), a technique similar to pulse oximetry, is based on obtaining a video of patient's fingertip. The flashlight of the smartphone lights up the patient's fingertip, and the camera detects the reflected color. As the heart contracts, the blood reaches the fingertip, and a video of the fingertip allows recording of the blood perfusion and analysis the color/signal intensity per unit of time (photoplethysmographic waves). ${ }^{9}$
When processing the signals obtained by a smartphone or a computer, it is possible to analyze the quantity and morphological characteristics of these pulse waves and to estimate heart rate, oxygen saturation, and blood pressure. ${ }^{9,11}$ Figure 1 shows how PPG technology works.

Schoettker et al., ${ }^{11}$ compared blood pressure measurements from a smartphone application that analyzes optical PPG signals (OptiBP) with blood pressure measurements by the conventional auscultatory method. After testing 50 patients, it was demonstrated that the OptiBP application is capable of accurately measuring blood pressure in an outpatient setting, making it an important tool for detecting hypertension.

Ridder et al., ${ }^{12}$ published a meta-analysis that included 14 studies that compared heart rate measured by applications and heart rate measured by equipment, and concluded that there was no significant difference between the results obtained by the smartphone and by commercial equipment, indicating a reliable use in adults. However, further studies are needed for children. Mitchell et al., ${ }^{13}$ analyzed 111 individuals who used Azumio ${ }^{\circledR}$ 's Instant Heart Rate apps on Android ${ }^{\circledR}$ and iOS ${ }^{\circledR}$ compared to an FT7 Polar ${ }^{\circledR}$ heart rate monitor and found an acceptable test-retest reliability at rest and post-exercise using the smartphone.

Nemcova et al., ${ }^{10}$ developed an Android ${ }^{\mathrm{TM}}$ application that used smartphone accessories, such as camera, flashlight, and microphone to predict heart rate, percentage of oxygen saturation $\left(\mathrm{SpO}_{2} \%\right)$, and blood pressure. Thirteen different smartphone models were used, and 65 signals from 22 individuals were obtained. Photoplethysmography was used to estimate heart rate and $\mathrm{SpO} 2 \%$. lood pressure was estimated using pulse transit time values. These were calculated by PPG and phonocardiogram (PCG), with cardiac sounds recorded by the smartphone placed on the chest, with a built-in microphone area pressed perpendicularly against to the cardiac auscultation point (Figure 2A). The App proved to be an alternative to estimate heart rate, $\mathrm{SpO}_{2}$, and blood pressure, indicating their relevance in mobile point of care Apps.

\section{Phonocardiogram with smartphone}

Cardiopathies such as heart valve diseases are often manifested by murmurs and sounds that can be heard during auscultation. ${ }^{14}$ Recently, a smartphone and a microphone were used to record sounds and heart murmurs to generate PCGs, which are graphs plotted with the variations of sounds over time. ${ }^{15}$ 


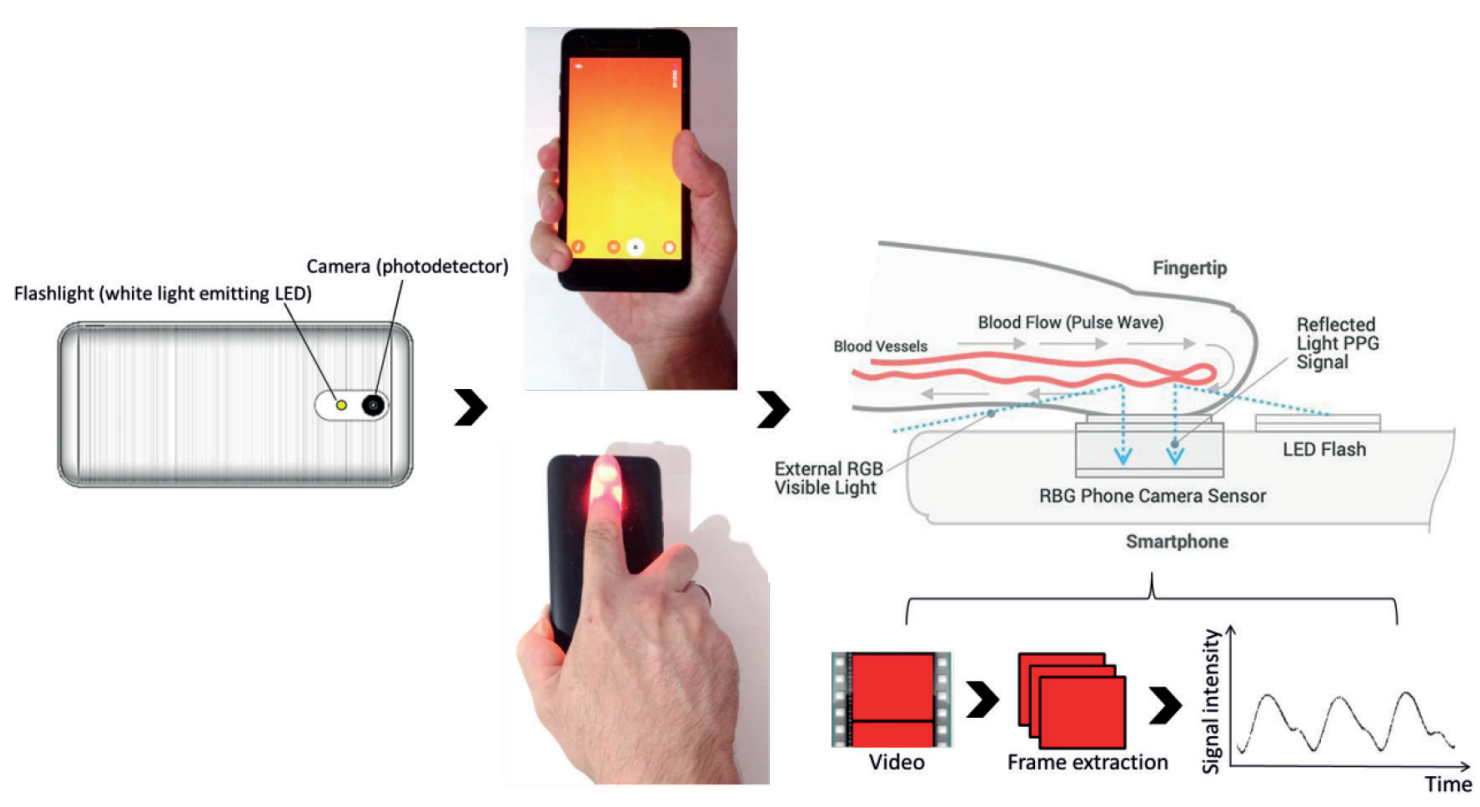

Figure 1 - A schematic detail of the smartphone-based photoplestimography technique. The flashlight functions as a light emitter, and the camera, as a photodetector. The light from the flashlight lights up the blood capillaries of the finger and the camera captures the reflected signal. Then, the frames are extracted from the videos and the signal is processed. Reproduced from Mazzu-Nascimento et al., ${ }^{8}$ and Schoettker et al., ${ }^{11}$ with permission from Creative Commons Attribution 4.0 International License.

It is possible to record cardiac sounds using stethoscopes attached to microphones or only microphones placed at the cardiac auscultation point to send the sounds to the smartphone for recording and processing the data. Thoms et al., ${ }^{15}$ were able to plot a PCG using the smartphone and a microphone through sound vibrations captured by the microphone. Researchers demonstrated that it is possible to determine heart rate and arrhythmias, but emphasized that background noises, breathing sounds, and noises produced by microphone motion can interfere with the quality of sound acquisition.

Kang et al., ${ }^{16}$ developed a smartphone android app (CPstethoscope) to perform cardiac auscultation using a smartphone and a built-in microphone to identify heart murmurs and physiological sounds. In 46 participants, three different smartphone models were used to capture the cardiac sounds recorded when the smartphone was placed at cardiac auscultation points on patient's chest (Figure 2A). Using a machine learning model, convolutional neural networks (CNNs) were used to process and classify sounds. Because the model only used the smartphone's built-in microphone, 16 sounds could not be interpreted due to capture failures. Of the 30 interpretable sounds, the classification accuracy was around $90 \%$, showing the viability of the app for cardiac auscultation.

Mamorita et al. ${ }^{17}$ developed a smartphone application capable of hearing and recording sounds and heart murmurs in real-time using a smartphone and an external microphone attached to a stethoscope. A simulator was used to generate the reference sounds (normal and pathological) and compare them with the sounds recorded by the smartphone. The results showed that the smartphone/microphone set obtained sound waves similar to the simulator, serving as an important tool to identify sounds and murmurs of the heart in real-time. Figure 2B shows the profiles of cardiac pathological sounds captured by the microphone.

\section{Electrocardiogram with smartphone}

The use of smartphones to perform an ECG can increase the accessibility to medical exams in even the most remote locations and a reduction of expenses when compared to the conventional exam. ${ }^{18}$

In the light of the pandemic caused by COVID-19, the Food and Drug Administration (FDA) authorized the use of the KardiaMobile-6L mobile device, produced by Alivecor, to detect atrial fibrillation. Giudicessi et al., ${ }^{19}$ pointed out 
A

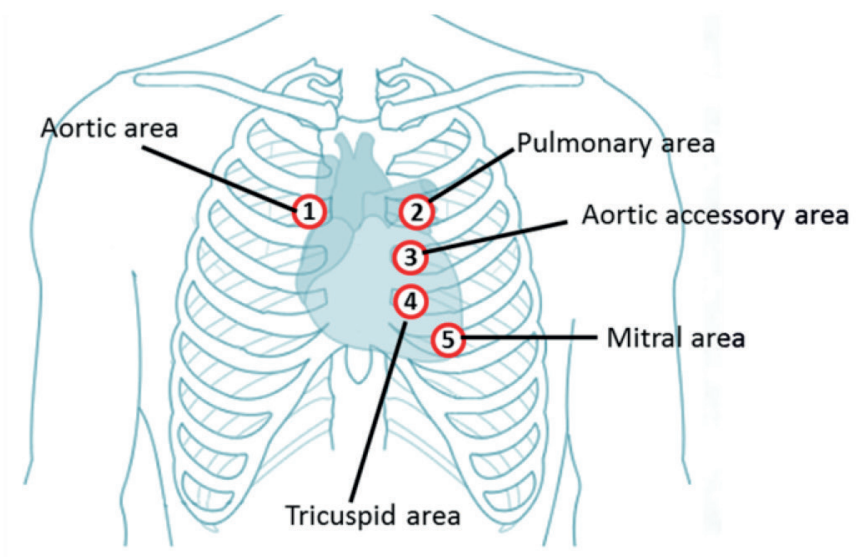

B

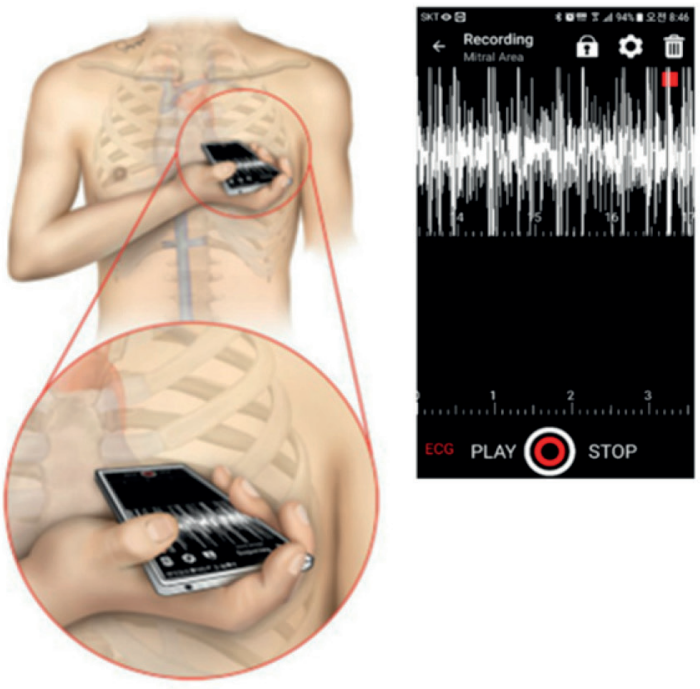

C

\begin{tabular}{l} 
Aortic stenosis \\
Mitral regurgitation \\
\hline Mitral stenosis \\
\hline Aubaortic stenosis \\
\hline Pulmonic stenosis \\
\hline Tentricular septal defect \\
\hline Acute mitral regurgitation \\
\hline Patent ductus arteriosus \\
\hline Mitral valve prolapse \\
\hline Dilated cardiomyopathy
\end{tabular}

Figure 2 - Capture of cardiac sounds using a microphone and smartphone. A) Auscultation points. B) Use of the smartphone's built-in microphone over the cardiac auscultation points. Reproduced from Kang et al. ${ }^{16}$ with permission from Creative Commons Attribution 4.0 International License. C) Profiles of sound characteristics of cardiac pathologies captured by the external microphone. Reproduced in part from Mamorita et $\mathrm{al}^{17}$ with permission from Karger Publishers.

the importance of using this model to remotely assess the cardiac condition of isolated patients diagnosed with COVID-19 to reduce the risk of contamination due to the lower exposure of health professionals. Also, the device does not require trained technicians to perform the exam. In this portable ECG device, the patient places his fingers on the device and in less than a minute, the ECG is obtained on the smartphone screen (Figure 3A).
Recently, in Canada, in a pilot study carried out in PHC, KardiaMobile devices were distributed to 184 doctors over a period of three months to investigate atrial fibrillation in 7,585 individuals. Atrial fibrillation was detected in 471 patients, showing an acceptable performance of the device in screening, and its potential as a clinical alternative in medical care in the future. ${ }^{20}$ 


\section{A}
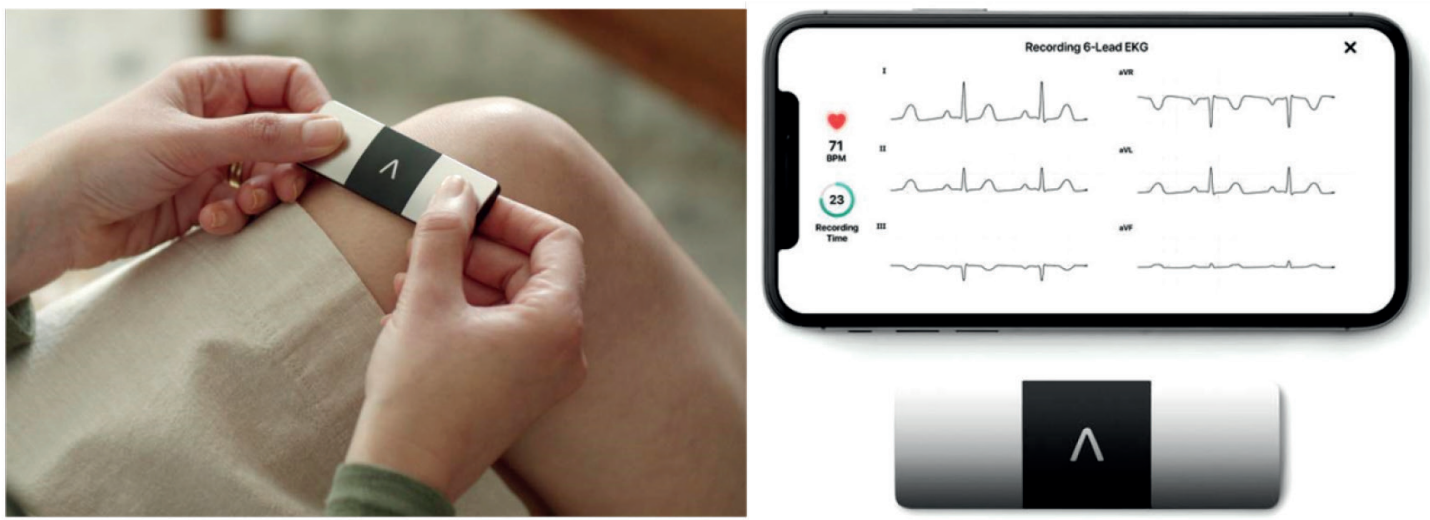

B
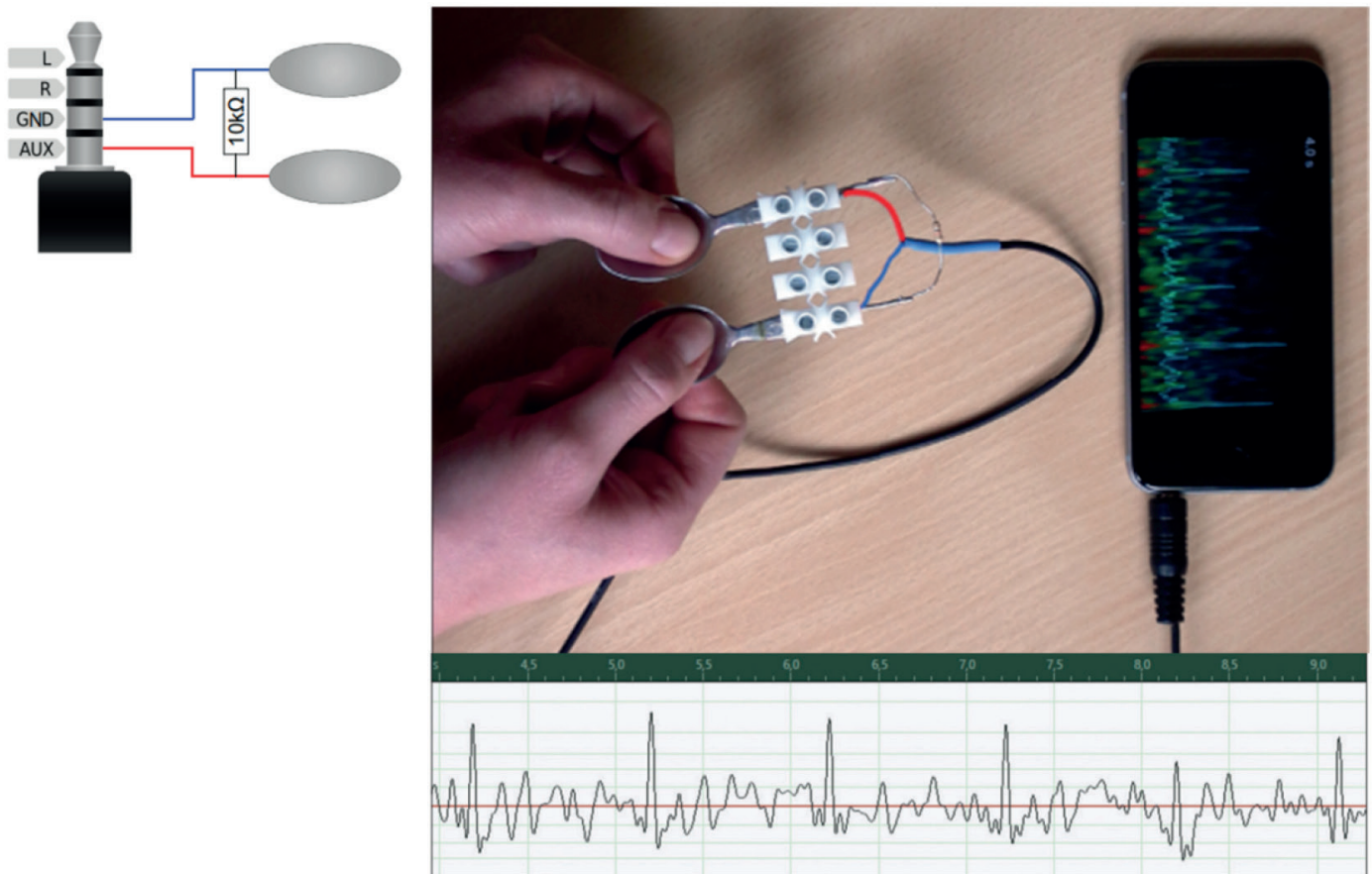

Figure 3 - Electrocardiograms obtained using a smartphone. A) KardiaMobile-6L commercial model used for remote monitoring of patients with COVID-19. Image reproduced with permission from AliveCor. B) Electronic scheme and electrocardiographic pattern generated by the low-cost sensor for electrocardiogram with the smartphone. Reproduced from Thoms et al., ${ }^{22}$ with permission from the American Association of Physics Teachers.

It is also possible to build an ECG device in a simple and low-cost way using electrodes, capacitors, and a smartphone, and a tutorial is available for this. ${ }^{21}$ As the cardiac impulse spreads through the heart, the electrical current also spreads from the heart to the surrounding tissues, reaching the body's surface.
Therefore, by attaching two electrodes on the skin surface on opposite sides of the heart, it is possible to capture the electrical potentials generated by the dipole field, ${ }^{22}$ similar to what happens with the Alivecor Kardia Mobile mobile device. Thoms el al., ${ }^{22}$ put this device into practice by using a cable and the 
earphone socket, two stainless steel plates functioning as electrodes making contact with the body, plus a $10 \mathrm{k} \Omega$ resistor connected in parallel with the input to reduce the polarization voltage. This circuit was tested on iPhone 6s and Samsung Galaxy S8, and it was possible to obtain the ECG at an experimental level (Figure 3B).

\section{Is it time to use artificial intelligence?}

Artificial intelligence (AI) is incorporated in software and functions like the human brain to make intelligent decisions, identify patterns, objects, and sounds, ${ }^{23}$ to encompass constant learning based on the most varied data sources through the use of algorithms, robotics, and artificial neural networks. ${ }^{24}$ Machine learning is part of the artificial intelligence approach inspired by human learning through examples and errors. A machine can recognize patterns and, at the end of the process, give a predictive answer to a complex question. One set of data is used to train the algorithms and another set to perform the tests to verify the model's accuracy. ${ }^{24,25}$

In cardiology, machine learning has been used in electrocardiography and echocardiography for automatic pattern recognition and classification of abnormal rhythms, interpretation of images, in nuclear cardiology to predict myocardial revascularization, among other approaches. ${ }^{26}$

Strategies to capture signals (image and sounds) from a smartphone's source have advanced rapidly, and several applications are capable of performing these tasks. It is noted that the biggest challenges in signal processing are eliminating noise, and improving prediction accuracy. ${ }^{27}$ Hence, it is necessary to explore different approaches to AI. Some of the techniques involving smartphones described above are already benefiting from AI.

Deep learning is a type of Machine Learning that learns through a taxonomy of functions distributed in layers, which allow meaningful learning of complex tasks. Different approaches using deep learning are being applied to filter out the noise and improve predictive power. Long Short-Term Memory (LSTM) networks employ a strategy based on forgetting long-term learning and have been very suitable for classifying, processing, and predicting time series. ${ }^{28}$ This approach has shown promising results in analyzing characteristics of ECG waves, which are temporal waves. ${ }^{29,30}$
A Brazilian study used more than two million ECG exams from 811 municipalities in the state of Minas Gerais using the Minas Gerais Telehealth Network (TNMG) for the automatic diagnosis of the 12-lead ECG using a deep neural network. It was demonstrated that Deep Neural Networks (DNNs) can accurately recognize anomalies such as bundle branch block, left bundle branch block, sinus bradycardia, atrial fibrillation, and sinus tachycardia more efficiently than medical doctors and residents. This approach shows that the use of DNN for ECG interpretation can increase the quality of the analysis and increase the population's access to diagnosis. ${ }^{31}$

Botina-Monsalve et al., ${ }^{32}$ used LSTM with a set of public data to filter PPG waves, to improve signal quality and increase the accuracy of heart rate measurements. Also, Liang et al., ${ }^{33}$ used a pre-trained CNN (GoogLeNet) to improve the classification and assessment of hypertension using PPG.

Recently, many studies have used two datasets with cardiac sounds to apply different processing techniques and classifications of cardiac sounds, the PhysioNet / CinC (2016) and the PASCAL (2011). ${ }^{34}$ The Short-time Fourier Transform (STFT), Wavelet transform, and Mel Frequency Cepstral Coefficients (MFCC) are widely used for the processing of cardiac sounds. The reason for processing a raw cardiac sound is to decompose a temporal signal (cardiac sounds over a period of time) into frequencies, to filter the sound and extract characteristics before classification. For classification, different machine learning approaches such as the Support Vector Machine (SVM) and the CNNs are being used for sound recognition and heart murmurs. ${ }^{35}$ Pre-trained CNN also serves as an option for classifying heart sounds. These networks are first trained with a dataset, then refinement and adjustments are applied to the layers of this pre-trained network. In addition, another dataset is used to train and perform the classifications. This strategy is very useful because deep-learning approaches need big data. Khan et al., ${ }^{36}$ used this approach by varying the use of the PhysioNet and PASCAL datasets and achieved an accuracy of $98.29 \%$ for classifying heart sounds.

Deep-learning has been recognized as a powerful approach that seems capable of providing the necessary accuracy to smartphones for screening cardiovascular diseases. The different architectures allow noise filtering, signal amplification, and increased predictive power. Considering the advances in mobile health, in the future, 
a multiparameter application can be created combining the analysis of different cardiovascular parameters to increase access to screening tests in underprivileged populations, such as people living in slums and rural areas with difficult access to health. A smartphone can hence analyze different signals, including PPG, PCG, and ECG to provide parameters such as heart rate, blood pressure, and $\mathrm{SpO}_{2}$, in addition to analyzing heart murmurs and electrical conduction of the heart. This app can be used directly in the population by trained personnel. People with urgent health needs can be referred to a hospital, and people with less serious health needs can be referred to PHC services. After the specialist care, patients can return to primary care facilities for health monitoring and treatment. Figure 4 illustrates this process.

A question that arises is: What is the effective way to handle big data in healthcare? The answer is by using cloud technology, since different algorithms can be implemented in the storage and processing of big data. Cloud technology in health has been used in the
COVID-19 pandemic ${ }^{37}$ and emerged as a possibility that could be implemented in health systems such as the Brazilian Unified Health System (SUS).

Considering the advances in the use of AI, it is important to consider the ethical aspects regarding the use of data, which can generate doubts regarding the responsibilities of the doctor and about the software. ${ }^{26}$ Nevertheless, technological tools are available to help but not replace clinical practice.

In Europe, on May 25, 2018, the new General Data Protection Regulation (GDPR) came into force, and in Brazil, there is the General Personal Data Protection Law (LGPD - Law No. 13.709 / 2018), which regulates the processing of personal data. ${ }^{38}$ With the action of $\mathrm{AI}$ and access to data for incorporation of these technologies, it is important to consider the security of this information. Authors have been suggesting improvements in data protection systems, especially in systems such as the Unified Health System (SUS) with the use of personal data stores (PDS), which would be owned and controlled by the user, who would hold an user identifier composed of an

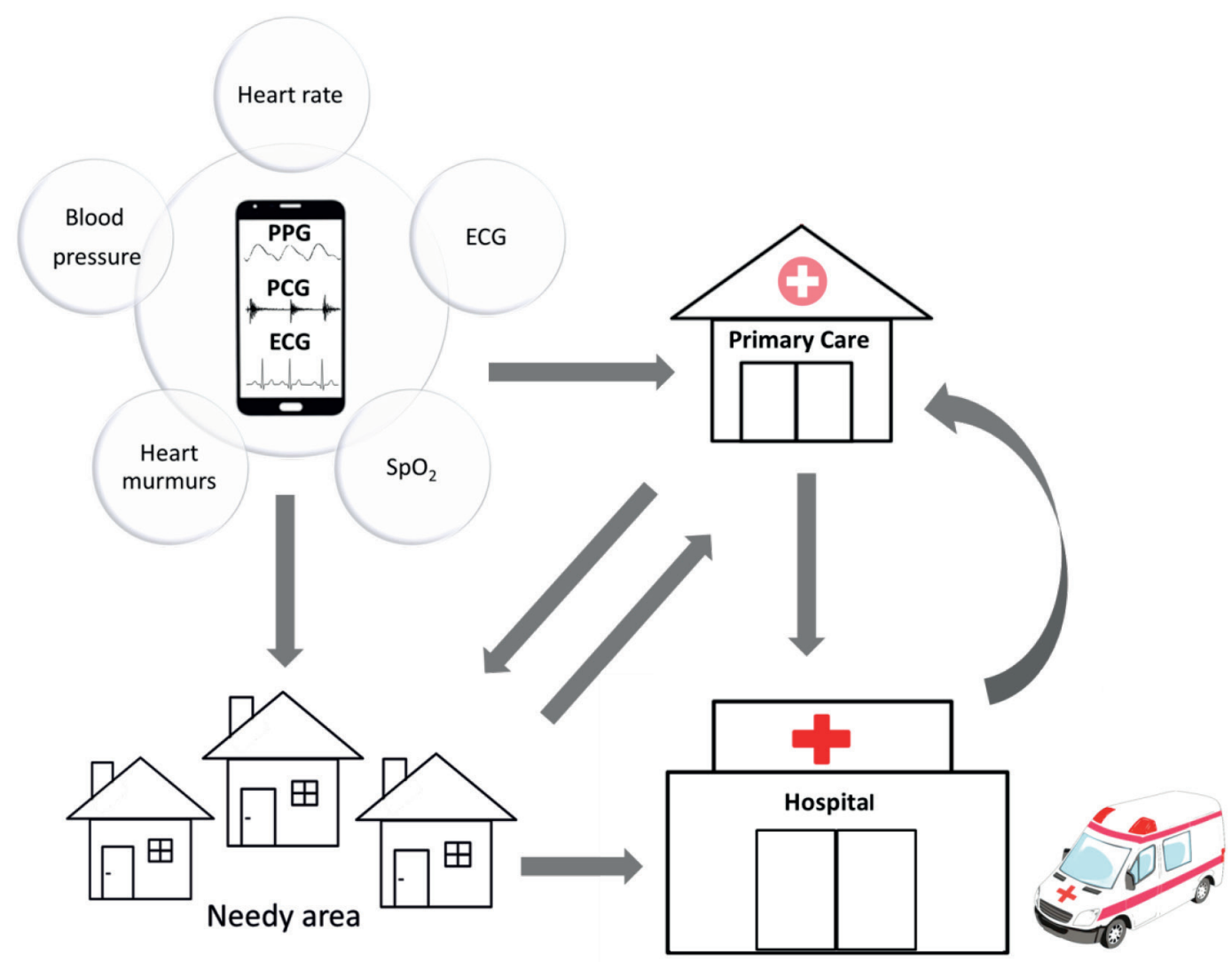

Figure 4 - Measurement of different cardiovascular parameters using a smartphone and flow of screening and care for a population in an underprivileged area. 
alphanumeric code to access exams. Despite the massive data access necessary for using AI, this does not seem to overshadow its incorporation in the health field..$^{39}$

\section{Final considerations}

Given the COVID-19 pandemic scenario and the growing demand for telemedicine and telecardiology solutions, it is essential to invest in health technology. Note the great potential of using smartphones in cardiology. The heart is a pump capable of working with three physical phenomena: optical, electrical, and acoustic. The techniques described here work exactly on the measurement of signals from these phenomena - cardiac frequency, blood pressure, and $\mathrm{SpO}_{2}$ (optical field), PCG (acoustic field), and ECG (electrical field). The use of smartphones for screening cardiovascular diseases is promising, however, advances in this area are necessary, including noise elimination, signal amplification, and improvement of predictive accuracy. The use of deep learning significantly improves the power of smartphones to analyze these parameters and appears as a fundamental technique to allow the use of smartphone in clinical screening on eliminating noise a large scale. The creation of applications or a multiparameter application proves to be very useful to increase access to people's health, especially in needy populations. We hope that in the near future the use of deep learning to improve mobile tools and development of multiparameter apps to enhance technology achievement will intensify.

\section{References}

1. World Health Organization (WHO). Fact Sheet Cardiovascular diseases (CVDs). [Internet]. 2017 May 2 [Last Update 2020 May 26]. Available from: https://www.who.int/news-room/fact-sheets/detail/ cardiovascular-diseases-(cvds)

2. Wernhart A, Gahbauer S, Haluza D. eHealth and telemedicine: Practices and beliefs among healthcare professionals and medical students at a medical university. PLoS One. 2019;14(2):1-13.

3. Lopes MACQ, de Oliveira GMM, Maia LM. Digital health, universal right, duty of the state? Arq Bras Cardiol. 2019;113(3):429-34.

4. Caetano R, Silva AB, Guedes ACCM, Paiva CCN, Ribeiro GDR, Santos DL, Silva RMD. Challenges and opportunities for telehealth during the COVID-19 pandemic: Ideas on spaces and initiatives in the Brazilian context. Cad Saude Publica. 2020;36(5):e00088920.

5. Chauhan V, Galwankar S, Arquilla B, Garg M, Somma SD, El-Menyar A, Krishnan V, Gerber J, Holland R, Stawicki SP. Novel Coronavirus (COVID-19): Leveraging Telemedicine to Optimize Care While Minimizing Exposures and Viral Transmission. J Emerg Trauma Shock. 2020;13(1):20-24

6. Mazzu-Nascimento T, Evangelista DN, Abubakar O, Souto BG, Domingues LV, Silva DF, et al. Remote and non-invasive monitoring of patients with COVID-19 by smartphone. Sci Med (Porto Alegre). 2021;31:e-39340.

\section{Author contributions}

Conception and design of the research: Mazzu-Nascimento T and Evangelista DN. Analysis and interpretation of the data: Mazzu-Nascimento T, Evangelista DN, Abubakar O. Writing of the manuscript: Mazzu-Nascimento T, Evangelista DN, Abubakar O, Roscani MG, Aguilar RS, Chachá SGF, da Rosa PR, Silva DF. Critical revision of the manuscript for intellectual content: Mazzu-Nascimento $\mathrm{T}$, Evangelista DN, Abubakar O, Roscani MG, Aguilar RS, Chachá SGF, da Rosa PR, Silva DF.

\section{Potential Conflict of Interest}

No potential conflict of interest relevant to this article was reported.

\section{Sources of Funding}

There were no external funding sources for this study.

\section{Study Association}

This study is not associated with any thesis or dissertation work.

\section{Ethics approval and consent to participate}

This article does not contain any studies with human participants or animals performed by any of the authors.

7. Madureira VHVG. Auto-atendimento de manutenção de terminais de pagamento: comparações entre realidade aumentada e vídeo. Tese. São Paulo: Fundação Getulio Vargas -(Gestão para Competividade); 2019.

8. Mazzu-Nascimento T, Evangelista DN, Abubakar O, Carrilho E, Silva DF, Chachá SGF, et al. Mobile Health (mHealth) and Advances in Noninvasive Diagnosis of Anemia: An Overview. Int J Nutrology. 2020;13(02):042-7.

9. Mazzu-Nascimento T, Leal ÂM de O, Nogueira-de-Almeida CA, de Avó LR da S, Carrilho E, Silva DF. Noninvasive Self-monitoring of Blood Glucose at Your Fingertips, Literally!: Smartphone-Based Photoplethysmography. Int J Nutrology. 2020;13(02):048-52.

10. Nemcova A, Jordanova I, Varecka M, Smisek R, Marsanova L, Smital L, et al. Monitoring of heart rate, blood oxygen saturation, and blood pressure using a smartphone. Biomed Signal Process Control. 2020;59:101928.

11. Schoettker P, Degott J, Hofmann G, Proença M, Bonnier G, Lemkaddem A, et al. Blood pressure measurements with the OptiBP smartphone app validated against reference auscultatory measurements. Sci Rep. 2020;10 (1):17827.

12. De Ridder B, Van Rompaey B, Kampen JK, Haine S, Dilles T. Smartphone Apps Using Photoplethysmography for Heart Rate Monitoring: MetaAnalysis. JMIR Cardio. 2018 Feb 27;2(1):e4. 
13. Mitchell K, Graff M, Hedt C, Simmons J. Reliability and validity of a smartphone pulse rate application for the assessment of resting and elevated pulse rate. Physiother Theory Pract. 2016;32(6):494-9.

14. Maganti K, Rigolin VH, Sarano ME, Bonow RO. Valvular heart disease: Diagnosis and management. Mayo Clin Proc. 2010;85(5):483-500.

15. Thoms LJ, Colicchia G, Girwidz R. Phonocardiography with a smartphone. Phys Educ. 2017;52. 023004.

16. Kang SH, Joe B, Yoon Y, Cho GY, Shin I, Suh JW. Cardiac Auscultation Using Smartphones: Pilot Study. JMIR Mhealth Uhealth. 2018 Feb 28;6(2):e49.

17. Mamorita N, Arisaka N, Isonaka R, Kawakami T, Takeuchi A. Development of a Smartphone App for Visualizing Heart Sounds and Murmurs. Cardiol. 2017;137(3):193-200.

18. Kidambi BR, Singal AK, Reddy SK. Smart Phone Electrocardiogram Bridging the Gap. J Pract Cardiovasc Sci. 2018;4(3):155-8.

19. Giudicessi JR, Noseworthy PA, Friedman PA, Ackerman MJ. Urgent Guidance for Navigating and Circumventing the QTc-Prolonging and Torsadogenic Potential of Possible Pharmacotherapies for Coronavirus Disease 19 (COVID-19). Mayo Clin Proc. 2020 Jun;95(6):1213-1221.

20. Godin R, Yeung C, Baranchuk A, Guerra P, Healey IS. Screening for Atrial Fibrillation Using a Mobile, Single-Lead Electrocardiogram in Canadian Primary Care Clinics. Can J Cardiol. 2019;35(7):840-5.

21. Tutorial for improved and more stable smartphone-ecg electrodes: https:// www.en.didaktik.physik.uni-muenchen.de/materials/ecgtutorial.pdf.

22. Thoms LJ, Colicchia G, Watzka B., Girwidz R. Electrocardiography with a Smartphone. Phys Teach. 2019;57(9):586-9.

23. Souza Filho EM, Fernandes FA, Soares CLA, Seixas FL, Santos AASMD, et al. Inteligência Artificial em Cardiologia: Conceitos, Ferramentas e Desafios - “Quem Corre é o Cavalo, Você Precisa ser o Jóquei." Arq Bras Cardiol. 2019:1-8. Doi: 10.36660/abc.20180431.

24. Smith LB, Slone LK. A Developmental Approach to Machine Learning? Front Psychol. 2017 Dec 5;8:2124.

25. Smyser CD, Dosenbach NU, Smyser TA, Snyder AZ, Rogers CE, Inder TE, Schlaggar BL, Neil JJ. Prediction of brain maturity in infants using machine-learning algorithms. Neuroimage. 2016 Aug 1;136:1-9.

26. Mesquita CT. Artificial Intelligence and Machine Learning in Cardiology A Change of Paradigm. Int J Cardiovasc Sci. 2017;30(3):187-8.
27. Baig MM, GholamHosseini H, Connolly MJ. Mobile healthcare applications: system design review, critical issues and challenges. Australas Phys Eng Sci Med. 2015;38(1):23-38.

28. Hochreiter S, Schmidhuber J. Long Short-Term Memory. Neural Comput. 1997; 9(8):1735-80.

29. Zhu F, Ye F, Fu Y, Liu Q, Shen B. Electrocardiogram generation with a bidirectional LSTM-CNN generative adversarial network. Sci Rep. 2019;9.6734. doi.org/10/1038/s41598-019-42516-Z

30. Abrishami H, Campbell M, Czosek R. Supervised ECG Interval Segmentation Using LSTM Neural Network. Int Conf Bioinforma Comput Biol BIOCOMP'18. 2018;(August):71-7.

31. Ribeiro AH, Ribeiro MH, Paixão GMM, Oliveira DM, Gomes PR, Canazart JA, et al. Automatic diagnosis of the 12-lead ECG using a deep neural network. Nat Commun. 2020 Apr 9;11(1):176.

32. Botina-Monsalve D, Benezeth Y, Macwan R, Pierrart P, Parra F, Nakamura K, et al. Long short-term memory deep-filter in remote photoplethysmography. 2020 IEEE/CVF Conference on Computer Vision and Pattern Recognition Workshops (CVPRW).2020.

33. Liang Y, Chen Z, Ward R, Elgendi M. Photoplethysmography and Deep Learning: Enhancing Hypertension Risk Stratification. Biosensors (Basel). 2018 Oct 26;8(4):101.

34. Chen W., Sun Q., Chen X., Xie G., Wu H., Xu C. Deep Learning Methods for Heart Sounds Classification: A Systematic Review. Entropy. 2021;23:667.doi: org./10.3390/e23060667

35. Tseng KK, Wang C., Huang YF, Chen GR, Yung KL, Ip WH. CrossDomain Transfer Learning for PCG Diagnosis Algorithm. Biosensors. April 2021;11(4). doi.org.10.3390/bios/1040127

36. Khan KN, Khan FA, Abid A, Olmez T, Dokur Z, Khandakar A, et al Deep learning based classification of unsegmented phonocardiogram spectrograms leveraging transfer learning. IEEE Access. 2020/12/15

37. Cresswell K, Williams R, Sheikh A. Using cloud technology in health care during the COVID-19 pandemic. Lancet Digit Heal. 2021;3(1):e4-5.

38. Henrique G., Lins A. Internet das Coisas e blockchain no Sistema Único de Saúde : a proteção dos dados sensíveis diante da Lei Geral de Proteção de Dados. Cad Ibero-Amer Dir Sanit. 2021;10(1):93-112.

39. Kfouri Neto M, Silva RDG., Nogaroli R. Inteligência Artificial E Big Data No Diagnóstico E Tratamento Da Covid-19 Na América Latina. Rev Bras Direitos Fundam Justiça. 2020;14(1):149-78. 